\title{
Characterization of Thermally Evaporated CdSe1-XSX Thin Films for Solar Cells Applications
}

\author{
M. Abdel-Rahman \\ Physics Department, Faculty of Science, Minia University, P.O. 61519, Minia, Egypt. \\ m_abdelrahman@mu.edu.eg
}

\begin{abstract}
Binary semiconductor CdSe and CdS thin films are widely used for optoelectronic devices and window materials. The formation of ternary $\mathrm{CdSe1-xSx}$ thin films improves the physical characteristics of the binary $\mathrm{CdSe}$ thin films. The importance of $\mathrm{CdSe1-xSx}$ thin film is the change of band gap when incorporating $\mathrm{S}$ into the CdSe. This change in energy gap recommends CdSe1-xSx thin film for photovoltaic and photoconductive cells applications. In this work, polycrystalline CdSe1-xSx thin films have been grown in terms of thermal evaporation technique. X-ray diffractometry has been used to determine the lattice parameters and the crystallite size of the $\mathrm{CdSe} 1-\mathrm{xS} x$ mixed crystals. The variation in lattice parameters with composition from $\mathrm{x}=0$ to $x=1$ were linearly. The crystallite size varies parabolically with the change in composition. The energy gap, $E_{g}^{o p t}$, values of CdSe1-xSx thin films were estimated in terms of first derivative of absorbance with respect to wavelength and found to be increased with the formation of the ternary compound Cd-Se-S and with increasing the $\mathrm{S}$ content as expense of Se. This wider energy gap of the prepared films, which permits extra light to reach the solar cell junction, was correlated with the change in the microstructure parameters of thin films. The electrical conductivity of $\mathrm{CdSe}_{1-x} \mathrm{~S}_{\mathrm{x}}$ thin films was studied and reveals two types of variation as a function of temperature. It was found that with increasing the sulfur content for both temperature ranges; an increase in the activation energy for all the prepared thin films has been observed.
\end{abstract}

Keywords: CdSe, CdS, CdSeS thin films, thermal evaporation, optical and electrical properties.

Subject Classification: Material Science

Language: English

Date of Publication: 30-06-2018

DOI: $10.24297 / j a p . v 14 i 2.7446$

ISSN: 2347-3487

Volume: 14 Issue: 2

Journal: Journal of Advances in Physics

Website: https://cirworld.com

This work is licensed under a Creative Commons Attribution 4.0 International License. 


\section{Introduction}

Semiconductor compounds II-VI thin films ( $\mathrm{CdSe}, \mathrm{CdS}, \mathrm{ZnTe}$, etc.) are characterized by their wide band gab [1] therefore; they have many scientific and technological applications. CdSe n-type semiconductor thin films with direct band gap ( $\mathrm{Eg}=1.7-1.8 \mathrm{eV}$ ) can be grown in cubic, hexagonal or polymorphism forms with high absorption coefficient and transmittance in the visible region. These CdSe films are widely used for optoelectronic devices fabrication [2] such as solar cells [3, 4], light emitting devices [5], lasers [6], photo detectors [7] and thin film transistors [8]. CdS thin films with band gap ( $\mathrm{Eg}=2.42 \mathrm{eV}$ ) [9] used extensively for heterojunction solar cells as for window material in connection with other semiconductors such as CdTe [10]. Short band gap width of the binary semiconductor affect negatively on the solar cells efficiency since nearly $20 \%$ of the incident photons will be absorbed by the semiconductor solar cell. Electrical and optical properties of semiconducting thin films are vital requirements for appropriate applications in several optoelectronic devices $[11,12]$. Modifying the composition of the ternary CdSe1-xSx thin films permits improvement of electrical, optical and structural properties of the binary CdSe thin films, therefore; widening of its scientific and technological applications [13]. Nano-sized CdSe1-xSx thin films can be prepared by several chemical and physical methods such as chemical bath deposition (CBD), chemical vapor deposition (CVD), RF-sputtering, spray pyrolysis, laser ablation and thermal evaporation techniques [13-16]. In the present work, Cd-Se-S has been synthesized as a mixture of the two systems $\mathrm{CdS}$ and $\mathrm{CdSe}$ by a chemical reaction method. The most well-known method, thermal evaporation technique, has been used to get the composition of CdSe1-xSx thin films. The structural, electrical and optical properties of the fabricated thin films have been investigated. The $\mathrm{X}$-ray diffraction technique has been used in analysis of the prepared thin films microstructure. Optical band gap has been deduced by the aid of the first derivative of absorbance with respect to wavelength. Electrical conductivity of $\mathrm{CdSe}_{1-x} \mathrm{~S}_{x}$ thin films was measured within temperature range of $300-450 \mathrm{~K}$ using four-probe method from which, the activation energy has been deduced from the conductivity variation with the reciprocal of absolute temperature.

\section{Experimental details}

Bulky polycrystalline CdSe1-xSx (with $x=0,0.250 .5,0.75$ and 1 ) were prepared using ball milling method as a conventional solid-state reaction technique. Stoichiometric amounts of $\mathrm{CdS}$ and CdSe powders with highpurity $(99.999 \%)$ obtained form Aldrich were mixed for about $30 \mathrm{~min}$ in a ball mortar consistent with the following reaction:

$$
x \mathrm{CdS}+(1-x) \mathrm{CdSe} \rightarrow \mathrm{CdSe} 1-\mathrm{xS} x
$$

The mixed powders pressed into a disk-shape pellet used as the starting constituents of the prepared semiconducting thin films. In our previous studies [17-21] different compounds has been prepared using the same technique. Using a conventional coating unit (Denton Vacuum DV 502 A) and at a pressure of about 10$6 \mathrm{~Pa}$, the compositions of $\mathrm{CdSe} 1-\mathrm{xS} x$ (with $0 \geq \mathrm{x} \geq 1$ ) thin films were deposited by thermally evaporating the powdered samples from a resistance heating quartz glass crucible onto the dried pre-cleaned glass substrates. The substrates were kept at constant temperature $(400 \mathrm{~K})$ and the deposition rate was adjusted at lower value $(2 \mathrm{~nm} / \mathrm{sec})$ to produce thin film compositions closely related to that of the bulk starting materials [21-23]. In order to obtain smooth and homogenous films, the substrates were rotated at slow speed of $5 \mathrm{rpm}$. The thickness of the produced films during evaporation process was monitored using thickness monitor FTM6. The as-deposited film thicknesses were studied at different compositions to avoid the film thickness effect on the prepared composition. The X-ray powder diffraction (XRD) Philips diffractometry (1710) with Cu-Ka radiation $(\lambda=1.54056 \AA)$ and $2 \theta$ between $5^{\circ}$ and $70^{\circ}$ with step-size of $0.02^{\circ}$ and step time of 0.6 seconds has been used to investigate the crystal structure and phase purity of the CdSe1-xSx (with $0 \geq x \geq 1$ ) thin films. The compositional analysis was carried out using energy dispersive $\mathrm{X}$-ray spectroscopy (EDAX). The relative error in determining of the indicated elements does not exceed $2.3 \%$. The absorbance optical spectra of the deposited thin films at room temperature were investigated using double beam spectrophotometer UV-VisNIR JASCO-670. 


\section{Results and Discussions:}

\subsection{X-Ray Diffraction analysis of CdSe1-xSx thin films}

The X-ray diffraction patterns for the $\mathrm{CdSe}, \mathrm{CdSe} 0.50 \mathrm{~S} 0.50$ and $\mathrm{CdS}$ powder with their characteristic peaks of reference code corresponding to each composition which exhibit polycrystalline nature are shown in Fig. 1. The XRD pattern of the CdSe1-xSx (with $0 \geq x \geq 1$ ) thin films deposited onto glass substrates are shown on Fig. 2. This figure shows that the diffraction planes (100) and (002) of the thermally evaporated CdSe1-xSx appeared for all prepared compositions at $x=0,0.25,0.50,0.75$ and 1 . Sharp diffraction line of the preferred crystalline orientation (002) reveals the good crystallinity with the wurtzite structure for all compositions between $\mathrm{CdSe}$ and CdS according to reference JCPDS code mentioned the inset of Fig. 1. Relative increase in the peak broadening of the XRD line (002) is observed with increasing the $x$ value (increasing the $S$ and decreasing the Se ratio) as shown in Fig. 3. This increase in the peak broadening maybe attributed to the variation of the interatomic spacing corresponding to the change in the crystalline phases from CdSe to CdS. There are significant outward shifts of the (002) peaks to larger diffraction angles occur gradually for films with incorporating $\mathrm{S}$ at expense of Se in the $\mathrm{CdSe} 1-\mathrm{xSx}$ thin films (clearly shown in Fig. 3.). This shift may be related to the smaller atomic radius $(1.09 \AA)$ of the $S$ atom than the atomic radius $(1.22 \AA)$ of the Se atom $[24,25]$.

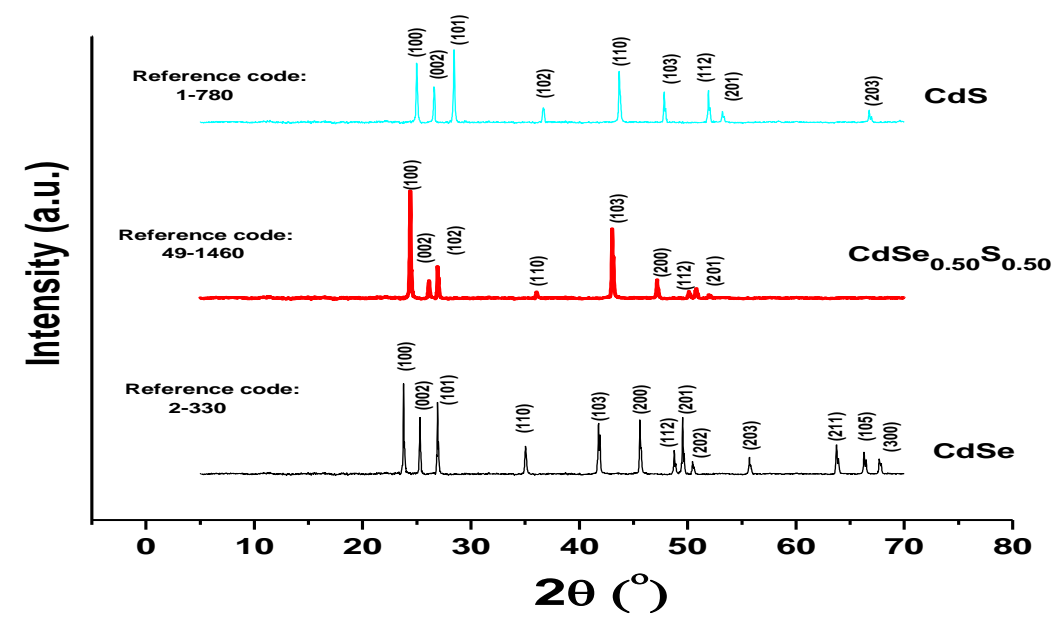

Fig. 1: XRD pattern of CdSe, CdSe0.50S0.50 and CdS powder.

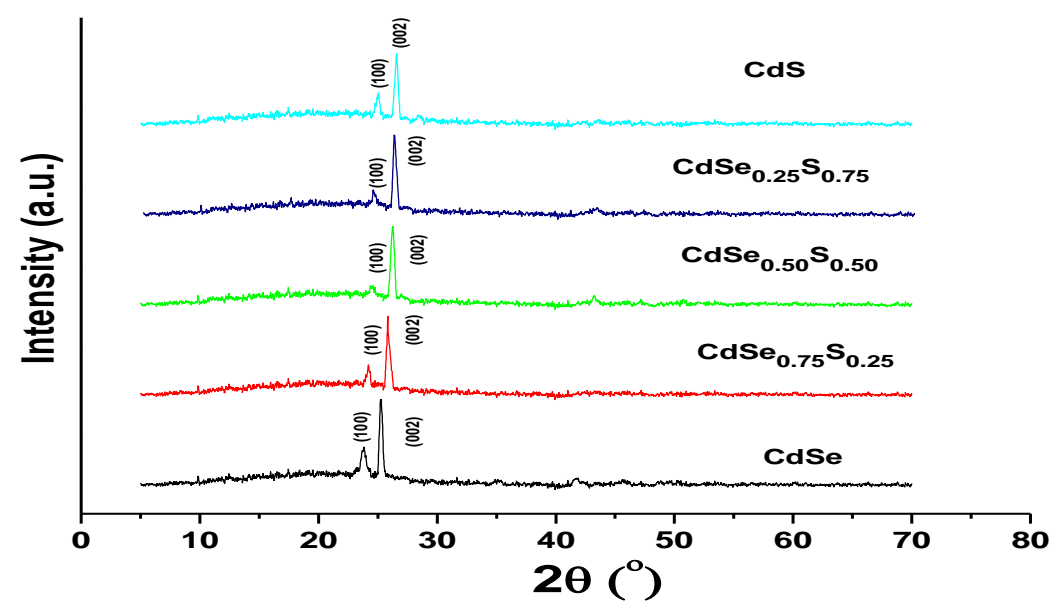

Fig. 2: XRD pattern of $\mathrm{CdSe}(1-\mathrm{x}) \mathrm{S} x$ thin films. 


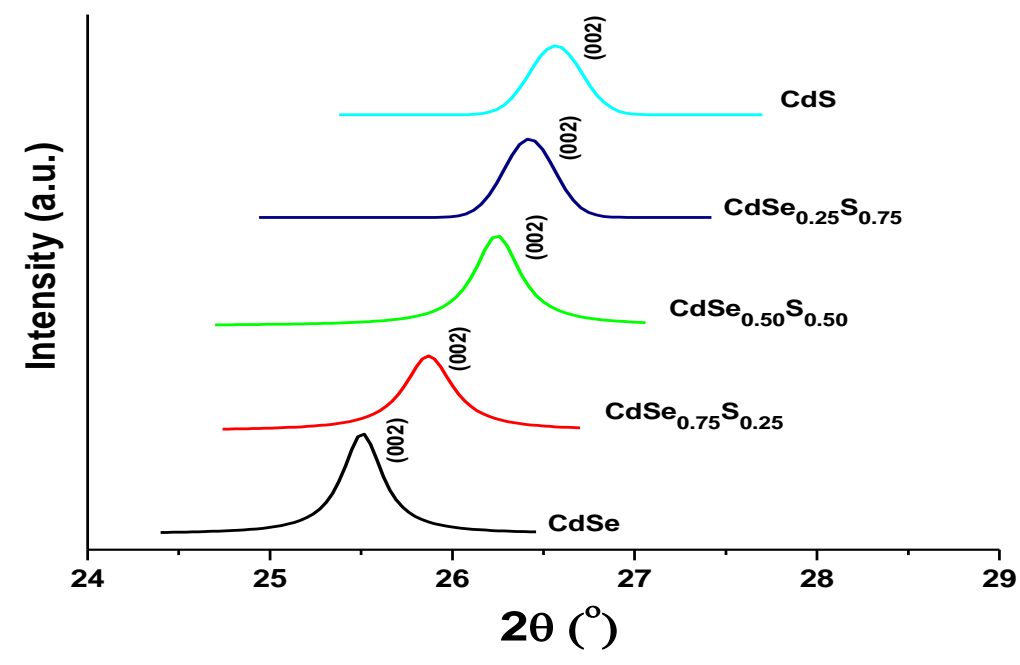

Fig. 3: XRD data related the variation of (002) with composition of $\mathrm{CdSe}(1-\mathrm{x}) \mathrm{Sx}$ thin films.

The interplaner spacing dhkl for these thin films were calculated from Bragg's law [26]

$$
\lambda=2 d_{h k l} \sin \theta
$$

where $\theta$ is the diffraction angle.

The hexagonal crystals are characterized by two lattice parameters, a and c, and the interplaner spacing dhkl given by [26]:

$$
\frac{1}{d_{h k l}^{2}}=\frac{4}{3} \frac{h^{2}+h k+k^{2}}{a^{2}}+\frac{l^{2}}{c^{2}}
$$

where hkl are Miller indices of the plane.
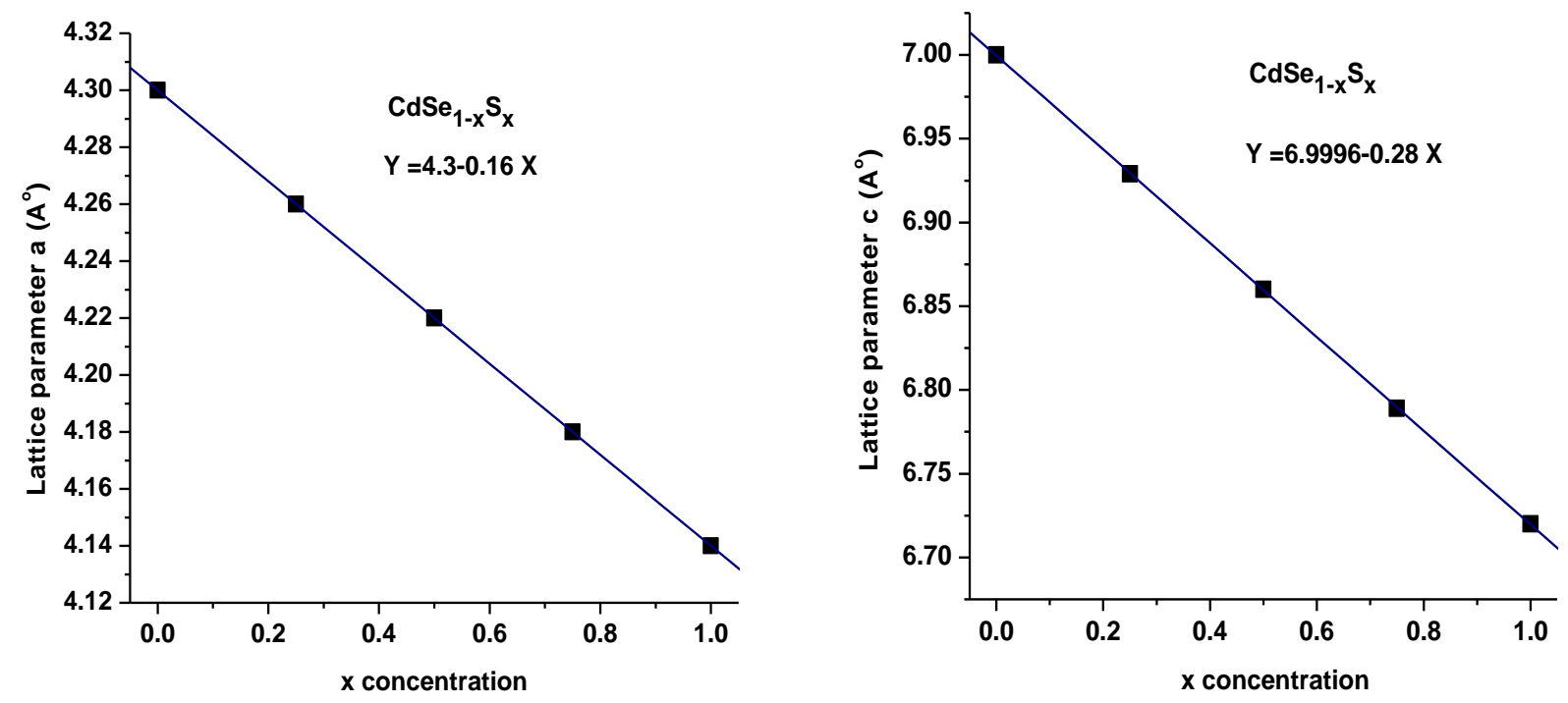

Fig. 4: Lattice parameters a and $\mathrm{c}$ as a function of composition 
The lattice parameters ( $a$ and $c$ ) were calculated and their variations with composition from $x=0$ to $x=1$ are shown in Fig. 4. The lattice parameters ( $a$ and $c$ ) decreased linearly with composition and seems to obey Vegard's [27]. The dependence of the lattice parameters a and c on composition has been found to obey the empirical relationships of the form: $a(x)=4.3-0.16 x$ and $c(x)=6.999-0.28 x$. Both microstructure parameters; crystallites size (Dv) and lattice strain (e) of CdSe1-xSx films were calculated by analyzing the XRD data using the Scherrer's formula, $D v=k \lambda / \beta \cos (\theta)$ and Wilson formula e $=\beta /(4 \tan (\theta)$ ) [28] respectively; where $\beta$ is the structural broadening, which is the difference in the integral $X$-ray peak profile width between the thin film and a standard (silicon) and is given by $\beta=\sqrt{\beta_{o b s}^{2}-\beta_{s t d}^{2}}$ [22, 23].

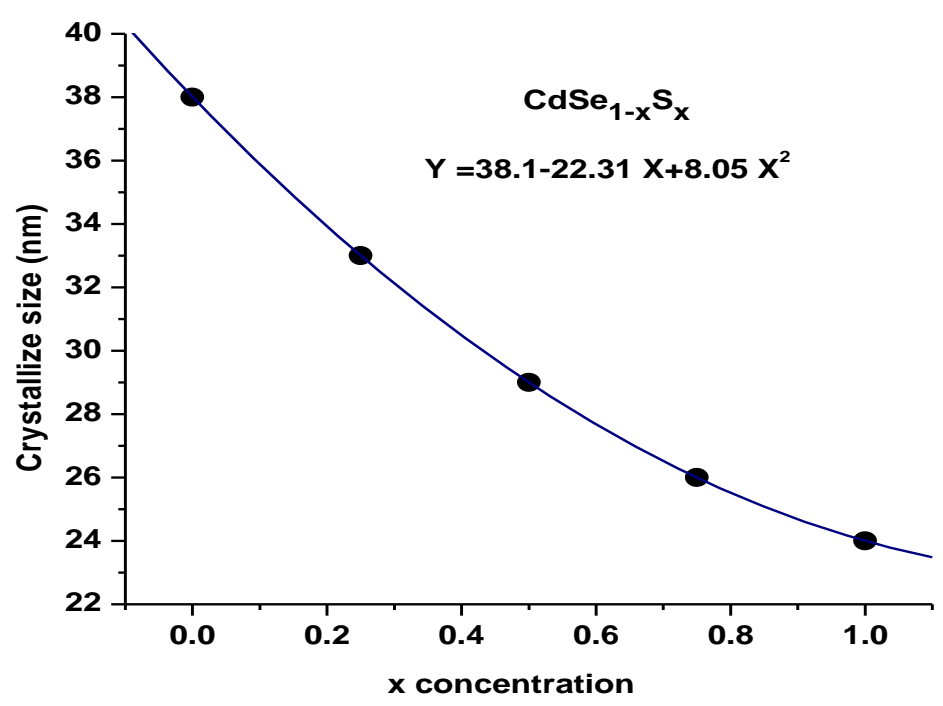

Fig. 5: Crystallite size as a function of composition.

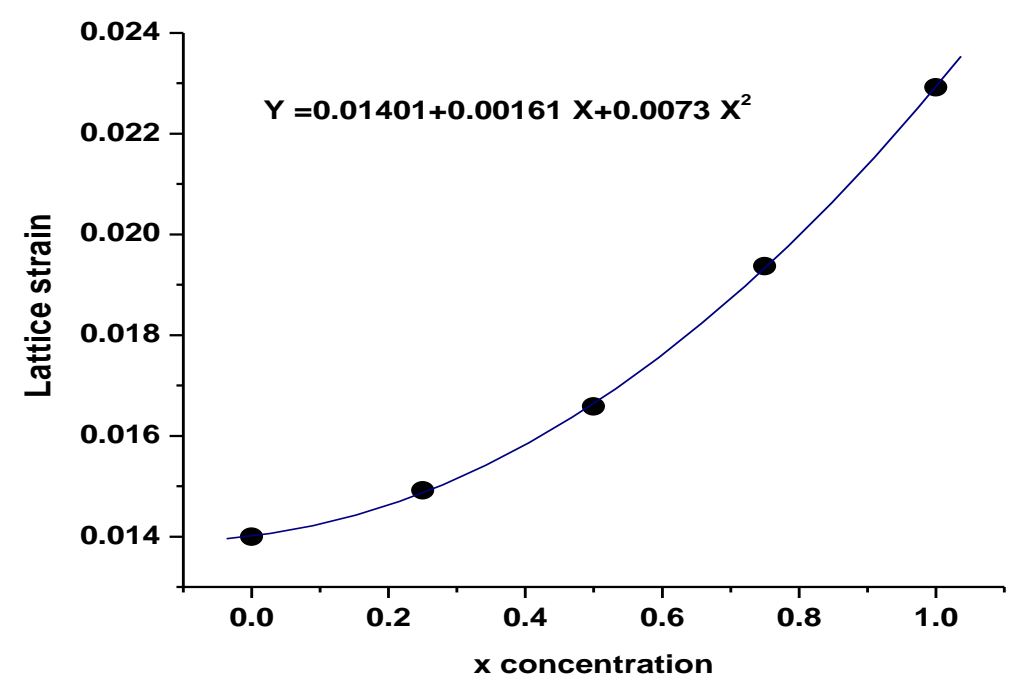

Fig. 6: Lattice strain as a function of composition.

Fig. 5 and Fig. 6 show a comparative look of the microstructure parameters (Dv and e) of the CdSe1-xSx films as a function of $x$ concentration. It is observed that the crystallite size decreases with increasing of $S$ at expense of Se as shown in Fig. 5 but the lattice strain ascends up slowly as shown in Fig. 6 . The decreasing in 
the crystallite size with $\mathrm{S}$ content may be attributed to the reduction in both lattice parameters $\mathrm{a}$ and $\mathrm{c}$ but the increasing in lattice strain maybe owing to increasing of lattice defects among the grain boundary, which might be attributed to the increase of breadth with increasing $S$ content.

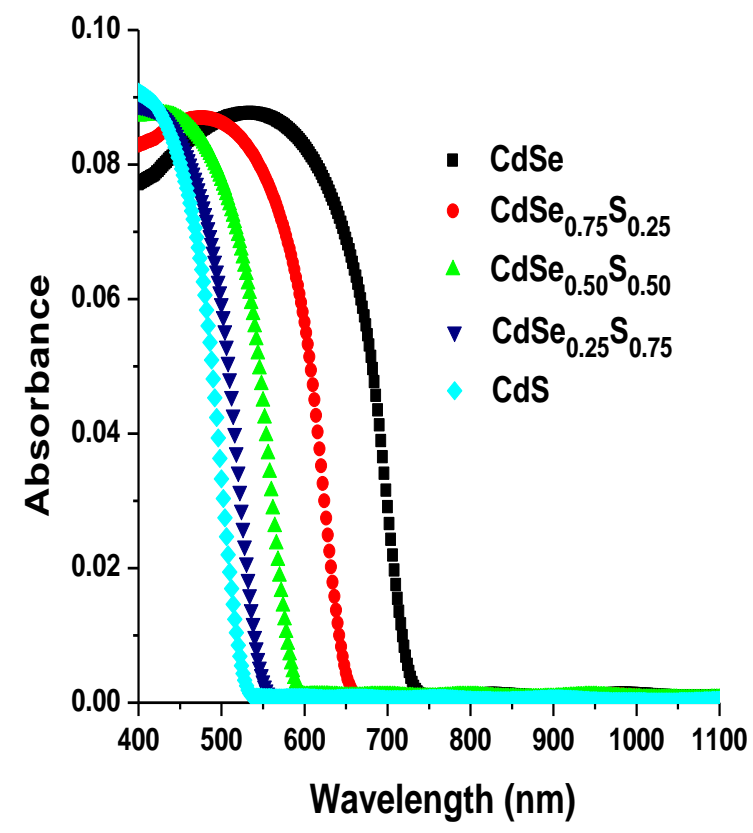

Fig. 7: Absorbance spectra of CdSe(1-x)Sx thin films.

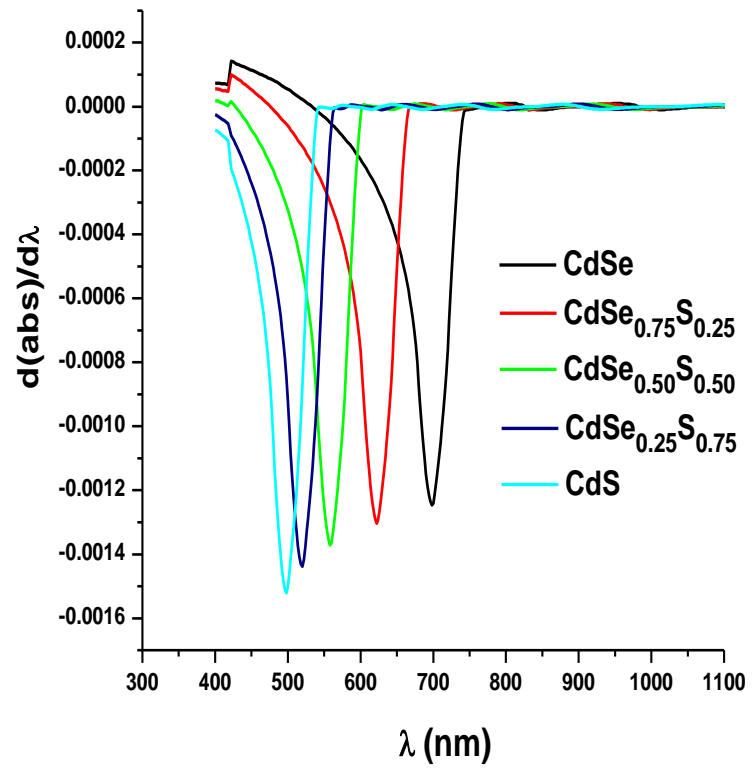

Fig. 8: the first derivative of absorbance as a

function of wavelength of the $\mathrm{CdSe}(1-\mathrm{x}) \mathrm{Sx}$.

\subsection{Optical absorbance and energy band gap of CdSe1-xSx thin films}

The double beam spectrophotometer was adopted to measure the absorbance in wavelength range extended from 400 to $1100 \mathrm{~nm}$ for different composition of CdSe1-xSx (with $0 \geq x \geq 1$ ) films as shown in Fig. 7. This figure shows that the absorbance shifted towards lower wavelength with increasing the $S$ at expense of Se. In order to extract the energy gap, $E_{g}^{o p t}$ value, first derivative of absorbance with respect to wavelength was considered. Fig 8 shows the differentiation of absorbance with respect to wavelength as a function of wavelength for $\mathrm{CdSe1-xSx}$ (with $0 \geq x \geq 1$ ). All the peaks that appear in this figure are due to the electronic transitions from fundamental band edge. The position of the peak has been used to estimate the band gap energy $E_{g}^{\text {opt }}$ for CdSe1-xSx (with $0 \geq x \geq 1$ ) $[29,30]$. It is also observed that the position of the peak shifts toward lower wavelength values with increasing the $S$ at expense of Se. 


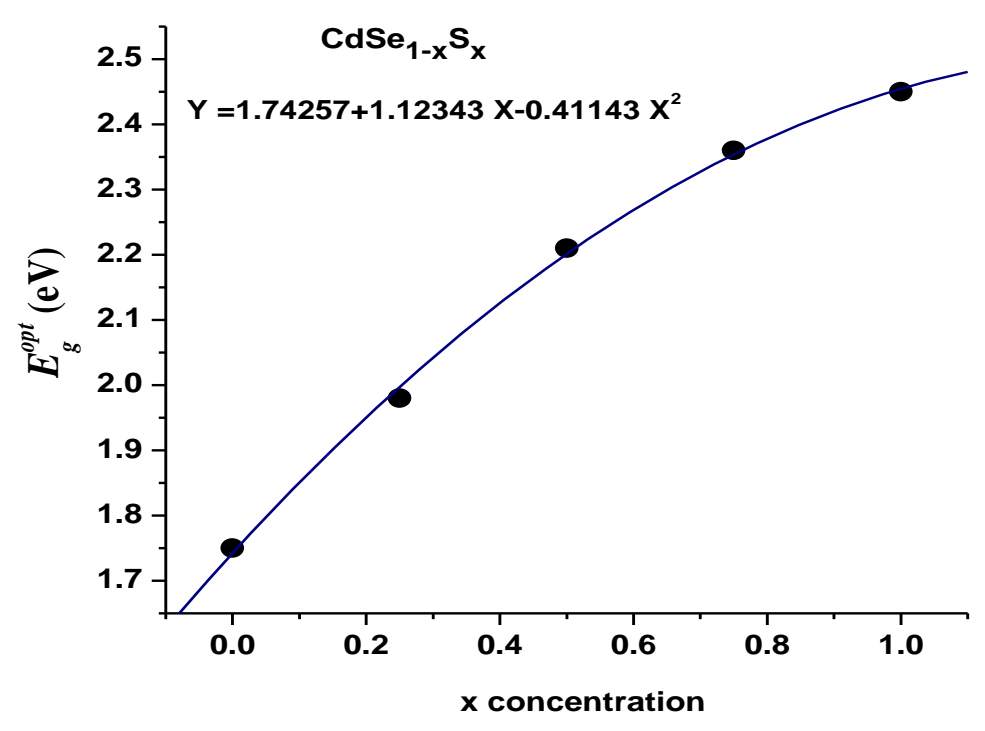

Fig. 9: Band gap energy as a function of the $x$ concentration in CdSe(1-x)Sx films.

The estimated optical band gap shown in Fig. 9 found to be increased with increasing the $S$ content for CdSe1-xSx thin film. The increasing in the optical band gap may be attributed to the following: (i) With increasing the $S$ content the particle size is decreases and owing to quantum confinement effects, the band gap energy increases. An incident photon will be absorbed by the semiconductor material only if the photon energy is the same as the energy gap of the material. The electronic band structure of the prepared nanoparticles changes to molecular level with non-vanishing energy spacing. So the nano scale size material needs more energy for electronic transition between valence and conduction band, hence the band gap energy of the prepared nanoparticles is more than that of the bulk material. (ii) Every particle in the nano scale size has very small number of atoms; the width of the bands gets narrower as the number of overlapping orbitals decreases, hence an increase in energy gap of the prepared nano-materials is observed. This tunability of the CdSe1-xSx band gaps recommends these thin film compositions as buffer layers for solar cells applications.

\subsection{Electrical conductivity of $\mathrm{CdSe}_{1-x} \mathrm{~S}_{\mathrm{x}}$ thin films}

The D.C. electrical conductivity of $\mathrm{CdSe}_{1-x} \mathrm{~S}_{\mathrm{x}}$ (with $\mathrm{x}=0,0.250 .5,0.75$ and 1 ) thin films at given temperature is given by Arrhenius relation [31- 33]:

$$
\sigma=\sigma_{0} e^{-\frac{\Delta E}{K T}}
$$

where $\sigma_{0}$ is a parameter depend on the nature of the semiconductor, $\Delta E$ is the thermal activation energy and $K$ is the Boltzmann constant.

Fig. 10 shows the variation of the D.C electrical conductivity as a function of the reciprocal of the absolute temperature at temperature range of $(300-450 \mathrm{~K})$ for $\mathrm{CdSe}_{1-\mathrm{x}} \mathrm{S}_{\mathrm{x}}$ thin films. This figure reveals the increasing behavior of the conductivity with temperature increase all over the temperature range. There are two different stages of the measured conductivity during the heating temperature range; display two different straight lines for every $\mathrm{CdSe}_{1-x} \mathrm{~S}_{x}$ composition as shown in Fig. 10. The first portion is the slow variation of the conductivity at low temperature range (300-370 K) and the conduction mechanism at this stage owing to carriers transport to localized (impurities) states near the valence and conduction bands. The second portion is the fast variation of the conductivity at high temperature range $(370-450 \mathrm{~K})$ and the conduction mechanism in this region due to the conduction of the carrier excited into the extended states beyond the mobility edge [21]. These two conduction mechanisms prove that the measured D.C. conductivity is non-linear with temperature change. The 
gradual decrease in the conductivity with excess of $\mathrm{S}$ content for five $\mathrm{CdSe}_{1-x} \mathrm{~S}_{x}$ compositions is also shown in Fig. 10. The main reasons for this gradual decrease in conductivity maybe related to: (i) The increase in the concentration of defects, such as vacancy of $\mathrm{Cd}$-shallow donor and (ii) The In- $\mathrm{CdSe}_{1-\mathrm{x}} \mathrm{S}_{\mathrm{x}}$ contact resistance at CdS content $\geq 50 \%$ (few $M \Omega$ ); contacts are not ohmic [34] (iii) Also, this decrease might be explained by the presence of the small size crystallites in the prepared $\mathrm{CdSe}_{1-x} \mathrm{~S}_{\mathrm{x}}$ thin films. The obtained results conclude that it is possible to use the models explaining the mechanism of electrical conduction in thin films with discrete structure for the prepared nanostructure polycrystalline samples [35]. It is also clear from Fig.10 that at low temperature region the electrical conductivity deviates from an Arrhenius behavior and changes in the conductivity with temperature are small. This suggests a variable range hopping conduction in this region by tunneling of the electrons between the localized states. This conduction mechanism can be verified by the Mott equation [36-38].

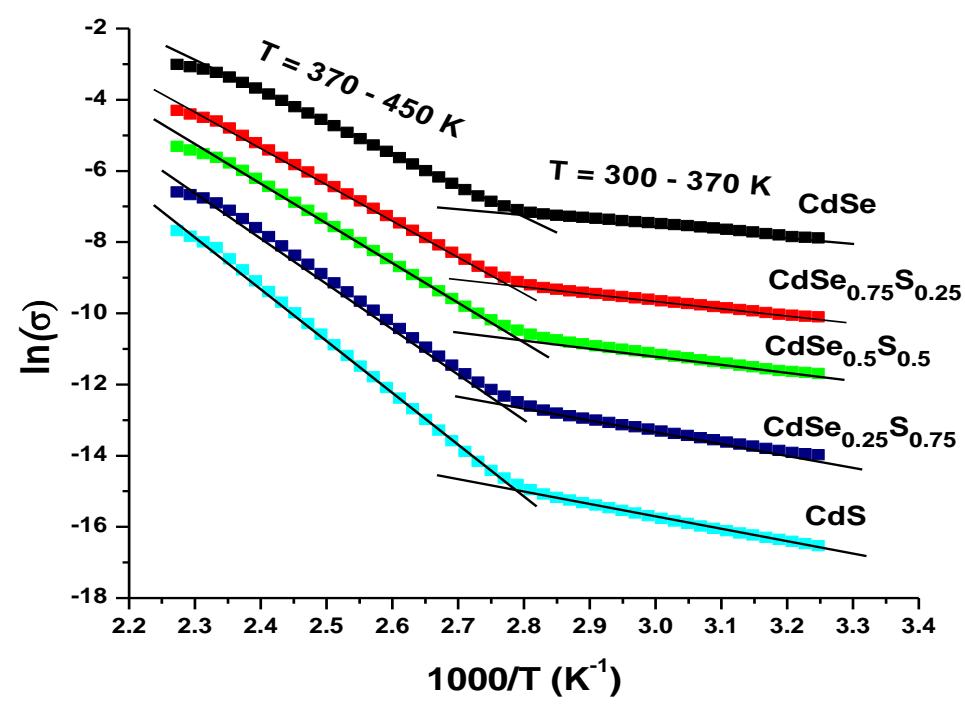

Fig. 10: Variation of electrical conductivity, $\sigma$ versus the reciprocal of absolute temperature for $C d \mathrm{Se}_{(1-x)} \mathrm{S}_{\mathrm{x}}$.

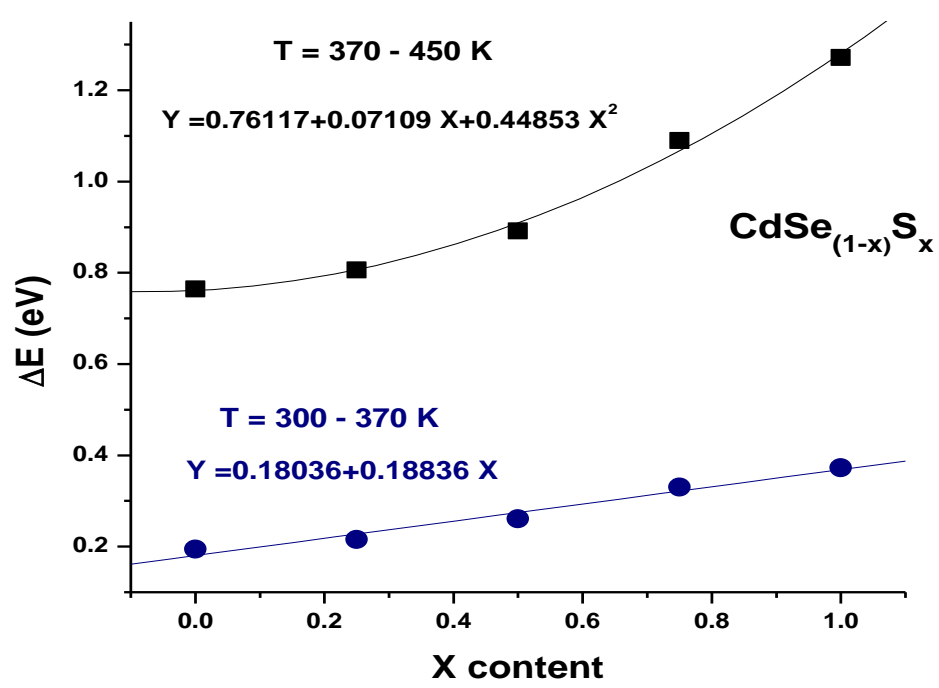

Fig. 11: Variation of activation energy of two portion region as a function of $x$ concentration. 
The activation energies as a function of the $x$ content in $\mathrm{CdSe}_{1-x} \mathrm{~S}_{\mathrm{x}}$ thin films of the two linear portions are shown in Fig. 11. The activation energy values for both stages show an increase with increasing $S$ content. In addition, the activation energies of the linear portion at low temperature range are lower than those at high temperature range. The obtained activation energy values are strongly differs from the band gap energies calculated by optical measurements for $\mathrm{CdSe}_{1-x} \mathrm{~S}_{x}$ thin films. This recommends that the determined activation energy values are associated with deep acceptor or shallow donor levels located in the forbidden band of the prepared $\mathrm{CdSe}_{1-x} \mathrm{~S}_{\mathrm{x}}$ thin films [39].

\section{Conclusions}

Polycrystalline powder of CdSe1-xSx $(x=0,0.250 .5,0.75$ and 1$)$ have been prepared by mixing CdSe and CdS powders using ball milling technique. The compositions of CdSe1-xSx (with $0 \geq x \geq 1$ ) have been fabricated as thin films by thermal evaporation technique. The CdSe1-xSx thin films have a wurtzite structure at which the lattice constants change linearly with composition over a wide range $(0<x<1)$. Both crystallite size and lattice strain were calculated using XRD. It is observed that the crystallite size decrease but the lattice strain increase with increasing $S$ at expense of $\mathrm{Se}$ in $\mathrm{CdSe1-xSx}$ films. The optical band gaps of all compositions were extracted from the differentiation of absorbance with respect to wavelength. The optical band gap increases with increasing the $\mathrm{S}$ content of the prepared composition. The increasing in energy gap was explained on the basis of decreasing particle size according to quantum confinement effects. The variation of the conductivity against reciprocal of the absolute temperature reveals two different straight lines related to extrinsic and intrinsic conduction. The activation energy values of the linear portion at low temperature range is lower than those at high temperature range and it increases with increasing the sulfur content in all films at both temperature ranges.

\section{Acknowledgments}

The author acknowledge helpfull assistance of Prof. Dr. E. R. Shaaban at the Physics Department, Faculty of Science, Al-Azhar University, Assiut, Egypt.

\section{References}

[1] S. M. Sze; "Semiconductor Devices: Physics And Technology" 2nd ed. John Wiley \& Sons, 1985.

[2] K. N. Shreekanthan, B.V. Rajendra, V. B. Kasturi and G. K. Shivakumar; Cryst. Res. Technol. 38, 30(2003).

[3] S. S. Kale and C. D. Lokhande; Materials Chemistry and Physics, 62 (2000) 103-108.

[4]Y. H. Yu, P. V. Kamat and M. A. Kuno; Adv. Funct. Mater. 20 (2010) 1464-1472.

[5] V. L. Colvin, M. C.Schlamp and A. P. Alivisatos; Nature, 370 (1994) 354-357.

[6] N. G. Zakharov, K. V. Vorontsov and Y. N. Frolov; Bulletin of the Lebedev Physics Institute., 42 (7) (2015) 216-220.

[7]Y. Jiang, W. J. Zhang, J. S. Jie, X. M. Meng; X. Fan and S. T. Lee; Adv. Funct. Mater. 17 (2007) 1795-1800.

[8] Jaeyoung Jang, Dmitriy S. Dolzhnikov, Wenyong Liu, Sooji Nam, Moonsub Shim and Dmitri V. Talapin; Nano Lett., 15 (2015) 6309-6317.

[9] A. Ashour; Turkish Journal of Physics, 27 No. 6 (2003) 551-558.

[10] K. D. Dobson, I Visoly Fisher, G. Hodes and D. Cahen; Solar Energy Materials and Solar Cells, 62 (2000) 295. 
[11] K. Sarmah, R. Sarma, H. L. Das; Chalc. Lett., 5 (2008) 153.

[12] N. J. Suthan kissinger, M. Jayachandran, K. Perumal, C. Sanjeevi raja, Bull. Mater. Sci. 30 (2007) 547.

[13] E. A. Sanchez-Ramirez, M. A. Hernandez-Perez, J. Aguilar-Hernandez, E. G. Palacios-Beas and M. Villanueva-Ibanez; Materials Research, 20 (4) (2017) 1121-1128.

[14] A.A. Yadav, M. A. Barote and M. A. Masumdar; Materials Chemistry and Physics, 121 (1-2) (2010) 53-57.

[15] M. A. Hrrnandez-Perez, J. Aguilar-Hernandez, G. Contreras-Puente, J. R. Vargas-Garcia and E. RangelSalinas; Physica E: Low-dimensional systems and Nanostructures, 40 (7) (2008) 2535-2539.

[16] A. S. Hassanien and A. A. Akl; Superlatice and Microstructures, 89 (2016) 153-169.

[17] E.R. Shaaban, M. El-Hagary, M. Emam-Ismail, A. Matar and I. S. Yahia; Materials Science and Engineering: B 178 (2013) 183.

[18] M. El-Hagary, A. Matar, E.R. Shaaban and M. Emam-Ismail; Materials Research Bulletin 48 (2013) 2279.

[19] M. Emam-Ismail, M. El-Hagary, E.R. Shaaban and S. Althoyaib; Journal of Alloys and Compounds 529 (2012) 113.

[20] M. El-Hagary, M. Emam-Ismail, E.R. Shaaban, A. Al-Rashidi and S. Althoyaib; Materials Chemistry and Physics 132 ( 2012) 581.

[21] E.R. Shaaban, A. Almohammedi, El Sayed Yousef, Gomaa A.M. Ali, Kwok Feng Chong, A. Adel and A. Ashour; Optik 164 (2018) 527-537.

[22] E.R. Shaaban, I. Kansal, S.H. Mohamed and J.M.F. Ferreira; Physica B 404(20) (2009) 3571-3576.

[23] E. R. Shaaban; J. Alloys Compd. 563 (2013) 274-279.

[24] R. G. Valeev, P.N. Krylov and E. A. Romanov; J. Surf. Invest. 1 (2007) 35-39

[25] K. M. M. Abo-Hassan, M.R. Muhamad and S. Radhakrishna; Physica B 358 (2005) 256-264

[26] B. Cullity; Elements of X-Ray Diffraction (Addison-Wesley, Reading, MA, 1976) pp. 1-80.

[27] O.P. Agnihotri and A.K. Raturi; Thin Solid Films 108 (1983) 313-317.

[28] B.D. Cullity \& S.R. Stock; Elements of X-Ray Diffraction, 3rd Ed., Prentice-Hall Inc., (2001) 167-171.

[29] B.G. Potter Jr and J.H. Simmons; Phys. Rev. B 37 (1988) 10838.

[30] R. Ramırez-Bon, F.J. Espinoza-Beltran, H. Arizpe-Chavez, O. Zelaya-Angel, and F. Sanchez-Sinencio, J. Appl. Phys. 77 (1995) 5461.

[31] A. F. Qasrawi and N. M. Gasanly; Semicond. Sci. Technol., 20 (2005) 446-452.

[32]. F. Yakuphanoglu, M. Sekerci and E. Evin; Physica B, 382 (2006). 21-25.

[33] A. S. Maan, D. R. Goyal, S. K. Sharma and T. P. Sharma; J. Phys. III France, 4, (1994). 493-501.

[34] G. V. Colibaba, E. V. Monaico, E. P. Goncearenco, D. D. Nedeoglo, I. M. Tiginyanu and K. Nielsch; Semicond. Sci. Technol. 29 (2014) 125003.

[35] G. Hurbecke; Ed. Polycrystalline Semiconductors, Physical Properties and Applications, Springer, Berlin (1985). 
[36] S.A. Lendave; Ph.D. Thesis, Solapur University, Solapur, M.S. India, 2011.

[37] L.P. Deshmukh; Ph.D. Thesis, Shivaji University, Kolhapur, M. S., India 1985.

[38] N.F. Mott and E.A. Davis; Electronic processes in non-crystalline materials, Oxford: Mott and Davis Clarendon Press (1971).

[39] R. B. Kale and C. D. Lokhande; Appl. Surf. Sci., 252, 929-938 (2005). 